\title{
Diagnostic evaluation and surgical management of recurrent hydatid cysts in an endemic region
}

\author{
Mehmet Yildirim ${ }^{1}$, Omer Engin ${ }^{1}$, Ozgür Oztekin ${ }^{2}$, \\ Fatih Akdamar', Zehra H Adibelli ${ }^{2}$ \\ ${ }^{1}$ Department of Surgery, ${ }^{2}$ Department of Radiology, \\ Izmir Bozyaka Teaching and Research Hospital, Izmir, Turkey
}

\begin{abstract}
Background. Echinococcosis is a zoonotic disease that occurs mainly in endemic areas. Recurrent hydatid cyst (RHC) and its diagnosis present insidious problems for surgeons. The aim of this study was to evaluate the diagnosis and surgical management of RHC in Turkey, where the disease is endemic.

Methods. We conducted a comprehensive study of 146 patients with abdominal hydatid cysts that were managed surgically between 1997 and 2007. Among those patients, RHC arose in 14 (9.5\%) of cases.

Results. The female to male patient-ratio was 6 to 8 and median age was 47.4 years. Patients' symptoms included abdominal pain, nausea, fever, and jaundice. Cysts reoccurred in patients sporadically between 4 months and 22 years. Abdominal US, CT, and MRI were utilized for the diagnosis in 14 (100\%), 11 (78.5\%), and $2(14.2 \%)$ patients respectively, demonstrating the RHC of the liver and concomitant cysts (splenic region, mesentery of the colon and perivesical) in all cases, respectively. The recurrent cysts (with a mean diameter of $12 \mathrm{~cm}$ ) were removed using partial pericystectomy, introflexion, and total cystectomy surgical techniques. Postoperative morbidity occurred in $14 \%$ of patients.

Conclusions. Especially in endemic countries like Turkey, RHC should be included in the differential diagnosis of cystic masses in abdominal organs that were previously treated for hydatid cysts. Surgery still remains the treatment of choice for RHC with early management and proper perioperative evaluation contributing to a high cure rate.
\end{abstract}

Key words: hydatid; cyst; recurrence

\section{Introduction}

Hydatid disease due to Echinococcus granulosus is endemic in particular regions of the world including the Mediterranean coun-

Received 31 March 2009

Accepted 1 July 2009

Correspondence to: Dr. Mehmet Yildirim, Atakent Mah. Bergama 2 Apt.Giris:32 Daire:1, Bostanl1/Izmir, Turkey. Phone: + 90232 3625692; Fax: + 90232 2614444; E-mail: mehmetyildi@gmail.com tries, the Middle East, Eastern Europe, South America, Australia and New Zealand. The clinical presentation of hydatid disease depends on the size and site of the lesion and the accessibility of the organ involved in the clinical examination. ${ }^{1}$

As there is no effective medical therapy for recurrent hydatid cyst (RHC), surgery is still the first-choice treatment. ${ }^{2}$ Administering pre- and post-operative courses of anti-helmintic drugs and using 


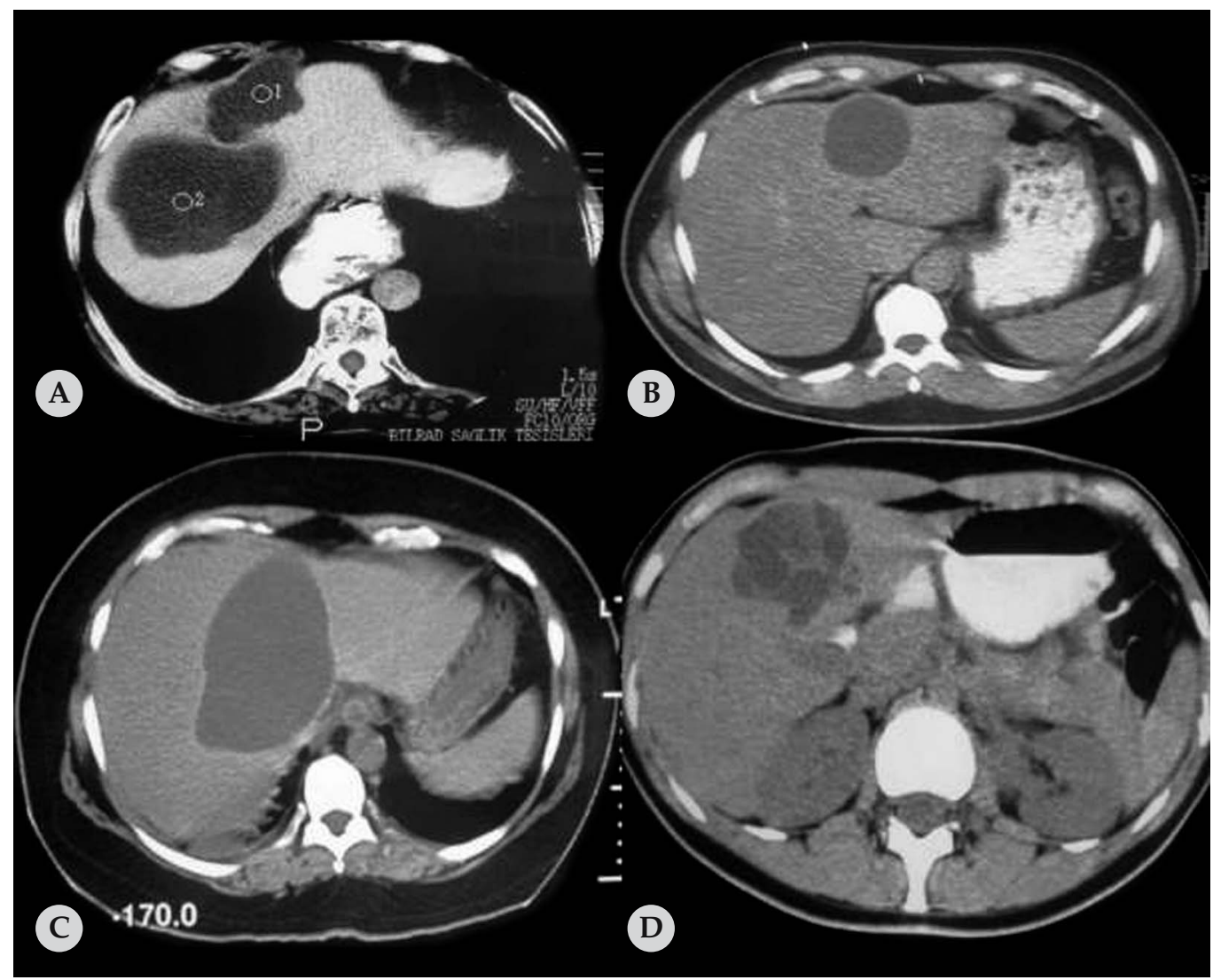

Figure 1. CT scan shows multiple (A) and uniloculer(B,C,D) RHC of the liver.

scolicidal solutions intra-operatively tends to kill the living daughter cysts, prevent the risk of spillage, and reduce the recurrence rate post-operatively. However, the recurrence rate of this disease is still disappointingly high, in the range of $9-20 \% .^{3}$

In our series of 146 patients with abdominal hydatid cysts that were managed surgically, a RHC arose in 14 (9.5\%) of cases. Since the recurrence of an echinococcal cyst mainly follows a silent clinical course, unless its growth produces symptoms, its diagnosis is commonly based on the findings of ultrasound (US), computed tomography $(\mathrm{CT})$, and magnetic resonance imaging (MRI). ${ }^{4}$

The aim of this study was to appraise within an endemic country the diagnosis and management of surgically confirmed abdominal RHC caused by E. granulosus at various locations in the abdomen.

\section{Patients and methods}

We, retrospectively, reviewed the medical records of 14 adult patients operated on for RHC at the Department of $2^{\text {nd }}$ Surgery at our Hospital (in Izmir, Turkey) during the period 1997-2007. We defined a 'recurrent cyst' to be any growing cyst at the original operative site, in the neighboring liver tissue, or extrahepatic. Our diagnostic workup for the study consisted of complete blood counts, liver function tests, hydatid serology with hemagglutination tests, and 
clinical findings. Imaging studies comprised US, CT and MRI. All patients underwent a preoperative US examination with $3 \mathrm{MHz}$ transabdominal probe. US findings were described according to Gharbi classification. ${ }^{5}$ Type 1 includes the univesicular cyst- pure fluid collection, Type 2 covers the fluid collection with a split wall-detached laminated membrane 'water lily' sign. Type 3 involves the fluid collection with septa and daughter cysts. Type 4 has a heterogenous appearence that Table 1. Time of previous surgery and location of the cysts

\begin{tabular}{lllll}
\hline Patient & Age & Sex & $\begin{array}{l}\text { Time of previous } \\
\text { surgery }\end{array}$ & Location of the RHC \\
\hline 1 & 48 & M & 22 years & liver \\
2 & 39 & F & 5-8 years & liver \\
3 & 37 & F & 8 years & liver \\
4 & 57 & M & $5-7-8-9$ years & Liver+mesentery \\
5 & 49 & M & $9-20-21$ years & liver \\
6 & 40 & M & 7 years & liver \\
7 & 57 & F & 3 years & Liver+perivesical \\
8 & 30 & M & $6-7-9$ years & liver \\
9 & 40 & F & 12 months & Liver+left \\
& & & & subphrenic fossa \\
10 & 52 & F & 15 months & liver \\
11 & 53 & M & 4 months & liver \\
12 & 47 & M & 15 months & liver \\
13 & 43 & M & 16 months & liver \\
14 & 39 & M & 5 years & liver \\
\hline
\end{tabular}
mimics a solid mass and Type 5 appears as reflecting thick walls and calcifications. CT examination was conducted for 11 patients with Toshiba spiral CT Asteion. MR studies were performed for two patients on a $1.5 \mathrm{~T}$ unit. The choice of the operative procedure was based on number, size, and location of cysts, and previous surgery. The pre- and post-operative one-month courses of anti-helmintic drugs (Albendazole) were administered to in all patients. The data were analyzed with a descriptive study.

\section{Results}

Fourteen of 146 of the patients in this study presented with RHC. The median age of 14 patients was 47.4 years (range, 30-64) at the time of diagnosis. Of the 14 patients, $6(42.8 \%)$ were females and $8(57.1 \%)$ were male.

Clinical symptoms varied and included abdominal pain $(n=14,100 \%)$, nausea $(n=$ $8,57.1 \%)$, fever $(n=2,14.2 \%)$, and jaundice $(n=1,7.1 \%)$. Hepatomegaly was noticed in

$8(57.1 \%)$ patients and an abdominal mass was detected in 2 (14.2\%). Cysts reoccurred in patients at irregular intervals between 4 months and 22 years. After their original operations, 5 patients experienced RHC less than 2 years later, 9 patients more than 2 years later (Table 1). Four patients had a recurrence of hydatid cyst between 5 and 21 years after one or more operations in their home hospital. The type of the initial operation performed was not known exactly. In one case, the same patient experienced recurrence twice, 5 and 8 years after the initial operation. Two patients had the surgical treatment for hydatid cyst three times. In one of those cases, cysts reoccurred 9, 20 , and 21 years after the original surgery. In the other, a recurrence arose 6, 7, and 9 years after the first operation. One case was operated four times: 5, 7, 8 and 9 years after three times surgery.

Eosinophilia, leukocytosis, and an increased level of ALT/AST were found in 1, 4 and 5 patients. The hemagglutination test was applied in $10(71.4 \%)$ patients and was positive in 4 of them. Abdominal 


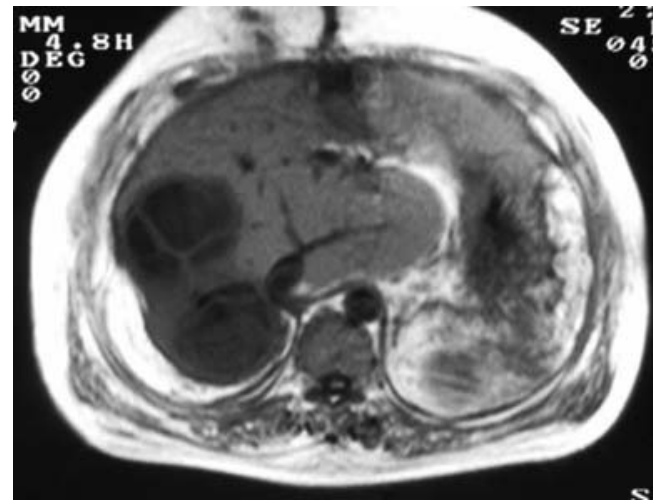

Figure 2. CT demonstrating the RHC of the liver and concomitant cyst of the splenic region. The patient was underwent splenectomy for splenic hydatid cyst in previous operation.

X-rays revealed an elevation of the right diaphragm in 3 (21.4\%) cases. Abdominal US, CT (Figure 1) and MRI were utilized in 14 $(100 \%), 11(78.5 \%)$, and $2(14.2 \%)$ patients, demonstrating the RHC of the liver in all patients. According to Gharbi classification, the majority of the cases were Types 1, 3 and $2(n=9,3$ and 2 cases). Three $(21.4 \%)$ patients from this group had associated hydatid cysts in other organs. The RHC was found in the left subphrenic space (Figure 2 ), the mesentery of the colon (Figure 3) and the perivesical region (Figure 4) in one patient. While one patient of the three had had a splenectomy as the treatment for a splenic hydatid cyst, the other two had been treated surgically for hydatid cyst of the liver, previously. Among 14 patients studied, the right lobe of the liver was affected in $11(78.5 \%)$ cases, the left lobe in $3(21.4 \%)$ cases. There were multiple liver cysts in $3(21.4 \%)$ cases. The mean diameter of the cysts was $12 \mathrm{~cm}$ (range 3.5 to $15 \mathrm{~cm}$ ). MRI was utilized successfully in 2 (14.4\%) patients, respectively (Figure 5).

In thirteen $(92.8 \%)$ of 14 patients studied, a subcostal incision was used. A midline incision was used for the remaining patient. At operation, we performed a complete removal of the contents of the hydatid cyst of

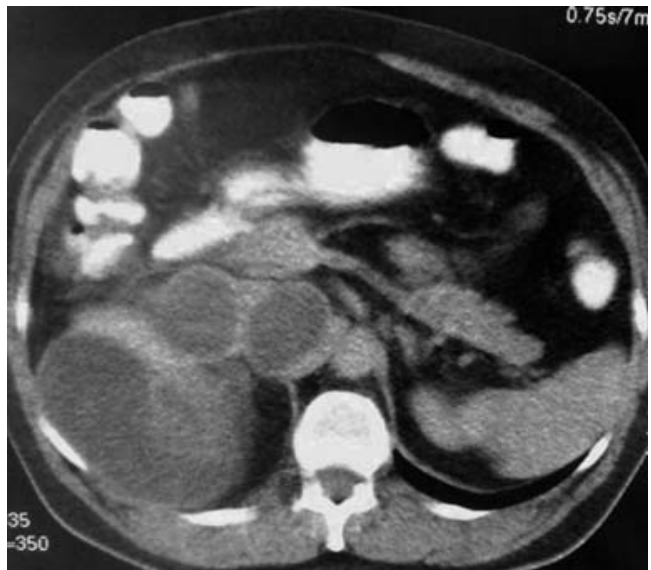

Figure 3. The patient was operated four times because of hydatid cyst. His CT scan shows RHC in the mesentery of the colon.

the liver in all 14 patients. We carried out partial pericystectomy and introflexion including the closure of the remaining cyst cavity to prevent dead space. The closure of the remaining cavity were drained and performed with silicon tubes. Total cystectomy was performed to the cyst of the colonic mesentery, the cyst in the splenic region, and the perivesical cyst. Postoperative morbidity was found in two (14.2\%) patients and consisted of wound infection. Only one patient was operated for brid ileus, 4 months later.

\section{Discussion}

In spite of modern therapeutic methods, RHC remains a significant problem for surgeons in endemic regions. A history of hydatid disease as well as the presence of its characteristic cystic lesions may well suggest the diagnosis of RHC, but it is often difficult to establish the unequivocal diagnosis before surgery. Although recurring cysts are commonly known to affect the liver and lung, studies also show that they can also affect other organs. ${ }^{6,7}$ Pre-operative diagnosis of RHC may be difficult, because 


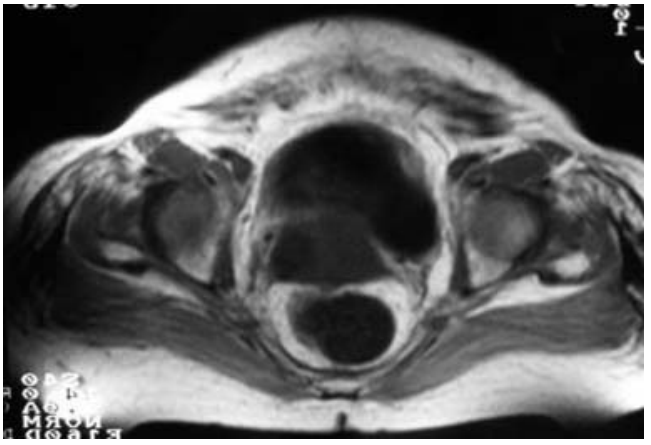

Figure 4. Perivesical location of the RHC in a 58-yearold woman.

it may be confused with primer or postoperative cysts (biloma,lymphocele). In our study, RHC was successfully diagnosed pre-operatively in all patients. The high success rate observed at our center could be explained by the referral of patients from endemic areas.

Unfortunately, a patient's prior history of hydatid cysts cannot in itself justify the diagnosis of the recurrent cyst. RHC develops most often as a new growth of an echinococcal cyst after the complete surgical removal of the primary one or due to a cyst developing postoperatively from the nonidentifiable small cysts. Another clinical scenario involves a cyst resulting secondarily to the surgical technique via disseminated vesicules, intraoperatively. In our study, we encountered RHC most likely due to the dissemination from the previous surgery in four patients.

The clinical presentation of RHC depends on the size and site of the lesion and the accessibility of the organ involved for the clinical examination. ${ }^{8}$ The cysts may become infected and present themselves as liver abscesses. This complication has been reported in the literature as occurring in 5\% to $40 \%$ of patients. ${ }^{9}$ In our experience, clinical symptomatology consists of abdominal pain, nausea, fever and jaundice. It appears that jaundice is related to the presence of a communication between a cyst and the bil-

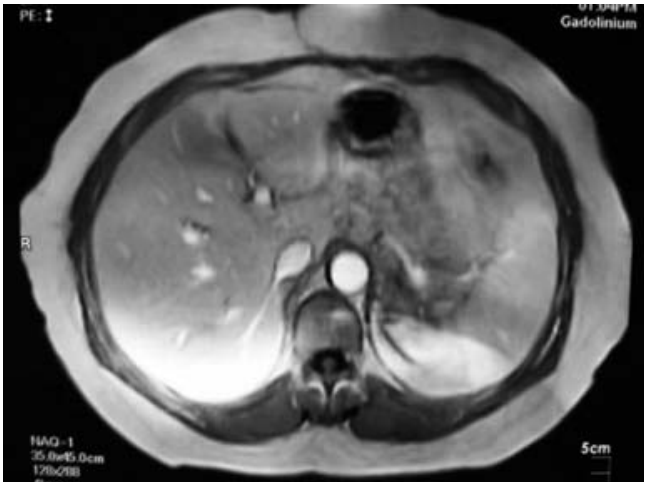

Figure 5. MRI appearence of the RHC on the left lobe of the liver.

iary tree although MRI did not confirm this supposition in our patients.

Although eosinophilia is expected in patients with parasitic infestations at the rate of $30 \%^{2}$, we only observed it in one $(7.1 \%)$ case. A previous study showed that hydatid immunoelectrophoresis, enzyme-linked immunosorbent assay (ELISA), and indirect hemagglutination (IHA) tests were conducted for the diagnosis and post-operative follow up. ${ }^{10}$ In our series of patients, serological tests identified cysts positively in 4 $(28.5 \%)$ of cases. The number of our serological tests is low due to the dead parasite and the impaired immune reaction.

Without having a patient's clinical history, pre-operative diagnosis of RHC cannot be made with imaging studies in asymptomatic and mildly symptomatic patients. A pre-operative diagnosis of RHC may be difficult, because it may be confused with a primer hydatid cyst or true cysts. As documented in this study, as well as others ${ }^{2}$, US and CT scans were the most helpful investigatory means for detecting abdominal cystic masses, successful in each of our 14 cases. US findings of the cysts may appear as purely cystic masses, completely detached membranes inside a cyst, multivesicular cysts, and cysts that are partially or totally calcified. CT findings of RHC include the round fluid collection in which membranes 
are detected, septa and cyst walls that are calcified, and primer hydatid cysts that are similar in appearance. Further, MRI, MRIangiography may be effective upon the visualization of suspected masses. ${ }^{11,12}$ In our study, all of the cysts were typed according to Gharbi classification with US. However, the classification of the cysts did not affect our surgical procedures. The treatment of the recurrent hydatid disease is mainly surgical, as anti-helmintic chemotherapy alone has failed in many cases. ${ }^{2}$ However, pre- and post-operative 1-month courses of anti-helmintic drugs should be considered in order to reduce the spillage of viable cysts during the surgery and to decrease the recurrence rate post-operatively. These drugs are recommended, as with our series of patients, for cases in which spillage of scoleces may have occurred during the surgery. A medical therapy may be used for patients with the inoperable disease, patients involved in the incomplete surgery, and for the prevention in patients of the secondary spread of echinococcal infection following the spontaneous rupture.

A surgical intervention seems to be a satisfactory approach to evacuate the contents of the cyst without spillage, and to prevent the cyst recurrence. ${ }^{13}$ At present, despite the ease of application, the percutaneous drainage of the RHC should be avoided, because it may lead to an intraperitoneal dissemination of the daughter cysts. The number and location of RHC, and their connection to adjacent structures, determine the surgical approach. The surgical intervention can be performed using either a transperitoneal or an extraperitoneal approach with a wide exposure of the abdominal cavity, since it demonstrates the overlooked cysts at the previous surgery. ${ }^{14}$ In our series, the subcostal incision was found to be effective, providing a good exposure for RHC within the liver.
Surgery may well be curative, but it carries risks including the secondary echinococcosis due to the spillage in $2 \%$ to $21 \%$ of cases. $^{2,15}$ Three key principles of the surgery are: aspiration of the cyst at its most superficial point, use of hypertonic saline solutions before opening the cavities (to kill the daughter cysts and prevent their further spread), and total cystectomy for extrahepatic locations intra-operatively. ${ }^{16}$ Liver cysts must be punctured with a canulla for the decompression and to determine the nature of intracystic fluid. In most of our cases we performed a partial pericystectomy / introflexion for liver cysts, and a total cystectomy for cysts at the other sites. When we review previously published articles, we find various types of treatment models for RHC. That implies that a proper and effective treatment modality is still not described. Although the surgery is the gold standard of RHC treatment, the effectiveness of the procedure is still quite questionable. What should be the surgical strategy to prevent RHC? For example, has the surgery removed all recurrence focus? Is spillage prevented during the operation? Are morphological changes, which might be the irregularities of the endocyst, creating pockets and preventing the contact between scolicidal agent and parasites? Has the RHC been correctly detected intra-operatively? Previous studies show that the resective surgery alone has been ineffective in preventing the cyst reoccurrence $-20 \%$ of such surgeries result in recurrences. But, a combined treatment method, resective surgery for macro disease and anti-helmintic drugs for the residual infection, is demonstrably more effective decreasing the recurrence rate to $10 \%$. Many authors recommend pericystectomy in the open surgery because it can be performed without opening the cyst, and without the spillage, and without the need for the cavity management. ${ }^{17}$ The limitations of the procedure is that it can only 
be performed on peripherally located small cysts and that it is associated with large amounts of blood loss.

RHC complications at recently reported rates of $10-37 \%$, in multiple series of patients treated surgically, include infection, hemorrhage, rupture, and obstruction of other abdominal organs. Additionally, reoperation rates have been reported as $6 \%{ }^{18}$ In our patients' series, laparotomy was performed in one patient, because of the brid ileus. Even though the mortality directly due to echinococcosis is rare, it can produce a very disabling morbidity. A mortality rate $1.5 \%$ has been reported. ${ }^{19}$

\section{Conclusions}

Surgical RHC failures may result in a new cyst(s) at a different location or in an undiscovered cyst(s) in a previous location. Since the prior history of the hydatid cyst cannot by itself justify the diagnosis of RHC, the rigorous evaluation of the patient is important for the improved diagnosis. An early diagnosis and the detection of small cysts are especially important - because if cysts can be detected and controlled at an early stage, they pose little or no threat pathologically or functionally to organs. Additionally, the proper perioperative evaluation and patients' support are efficacious in improving surgical results. We recommend a total or partial pericystectomy as the most appropriate procedure for the management of primary $\mathrm{HC}$ and also for RHC. Furthermore, we advocate RHC incidence may be decreased with the improvement of misdiagnoses, mistreatment, and appropriate follow-up and also the preventive immunologic research in the biology of the parasite.

\section{References}

1. Atalay F, Kirimlioglu V, Gundogdu H, Akincioglu T, Gencer A. Surgery for hydatid cysts of the liver. Hiroshima J Med Sci 1995; 44: 89-92.

2. Safioleas M, Misiakos E, Manti C, Katsikas D, Skalkeas G. Diagnostic evaluation and surgical management of hydatid disease of the liver. World J Surg 1994; 18: 859-65.

3. Akyildiz HY, Akcan A, Karahan I, Kucuk C, Sözüer E, Esin H. Recurrent liver hydatid disease: when does it become symptomatic and how does one diagnose it? Clin Imaging 2009; 33: 55-8.

4. Sielaff TD, Taylor B, Langer B. Recurrence of hydatid disease. World J Surg 2001; 25: 83-6.

5. Gharbi HA, Hassine W, Brauner MW, Dupuch K. Ultrasound examination of the hydatic liver. Radiology 1981; 139: 459-63.

6. Chokki A, Zribi R, Nouira S, Dziri Ch. Prevesical hydatid cyst: An exceptional occurrence. J Postgrad Med 2008; 54: 313-5.

7. Bal N, Kocer NE, Arpaci R, Ezer A, Kayaselcuk F. Uncommon locations of hydatid cyst. Saudi Med J 2008; 29: 1004-8.

8. Avgerinos ED, Pavlakis E, Stathoulopoulos A, Manoukas E, Skarpas G, Tsatsoulis P. Clinical presentations and surgical management of liver hydatidosis: our 20 year experience. IHPBA Journal HPB 2006; 8: 189-93.

9. Manterola C, Barroso M, Vial M, Bustos L, Muñoz $\mathrm{S}$, Losada $\mathrm{H}$, et al. Liver abscess of hydatid origin: clinical features and results of aggressive treatment. ANZ J Surg 2003; 73: 220-4.

10. Farmer PM, Chatterley S, Spier N. Echinococcal cyst of the liver: diagnosis and surgical management. Ann Clin Lab Sci 1990; 20: 385-91.

11. Aschauer MA, Stollberger R, Ebner F. Advances in contrast-enhanced MR-angiography: indications and limitations. Radiol Oncol 2002; 36: 103-8.

12. Heikal AA, Wachowicz K, Thomas SD, Fallone BG. A phantom to assess the accuracy of tumor delineation using MRSI. Radiol Oncol 2008; 42: 232-9.

13. Cangiotti L, Giulini SM, Muiesan P, Nodari F, Begni A, Tiberio G. Hydatid disease of the liver: long term results of surgical treatment. G Chir 1991; 12: 501-4. 
14. Gollackner B, Langle F, Auer H, Maier A, Mittlbock M, Agstner I, et al. Radical surgical therapy of abdominal cystic hydatid disease: factors of recurrence. World J Surg 2000; 24: 717-21.

15. Lucey BC, Kuligowska E. Radiologic management of cysts in the abdomen and pelvis. Am J Radiol 2006; 186: 562-73.

16. Kune GA, Schellenberger R. Current management of liver hydatid cysts: results of a 10-year study. Med J Aust 1988; 149: 26-30.

17. Belli L, Aseni P, Rondinara GF, Bertini M. Improved results with pericystectomy in normothermic ischemia for hepatic hydatidosis. Surg Gynecol Obstet 1986; 163: 127-32.

18. Prousalidis J, Kosmidis C, Anthimidis G, Fachantidis E, Harlaftis N, Aletras H. Forty-four years' experience (1963- 2006) in the management of primarily infected hydatid cyst of the liver. IHPBA Journal HPB 2008; 10: 18-24.

19. Safioleas M, Misiakos EP, Kakisis J, Manti C, Papachristodoulou A, Lambrou P, et al. Surgical treatment of human echinococcosis. Int Surg 2000; 85: 358-65. 